\title{
The Tendinous Impaction- Fibroma of Tendon Sheath
}

\author{
Anubha Bajaj \\ Consultant Histopathologist, A.B. Diagnostics Delhi, India \\ *Corresponding author: Anubha Bajaj, Consultant Histopathologist, A.B. Diagnostics Delhi, India. \\ Received date: July 7 2020; Accepted date: July 20, 2020; published date: July 22, 2020 \\ Citation: Anubha Bajaj. The Tendinous Impaction- Fibroma of Tendon Sheath. J Clinical Research and Reports, 5(1); DOI:10.31579/2690-1919/104 \\ Copyright: () 2020 Anubha Bajaj. This is an open access article distributed under the Creative Commons Attribution License, which permits \\ unrestricted use, distribution, and reproduction in any medium, provided the original work is properly cited.
}

Preface Fibroma of tendon sheath (FTS) was initially scripted by Geschickter et al in 1949. Subsequently, Chung and Enzinger elaborated upon cogent clinical and pathological manifestations of the neoplasm(1).

Fibroma of tendon sheath is denominated as a benign, fibroblastic, nodular neoplasm which emerges from synovium of tendon sheath. As fibroma of tendon sheath characteristically originates from adherent tendons and tendon sheaths, it predominantly incriminates small joints of upper limb as fingers, hands and wrists. Infrequently, the toe, foot, ankle, leg, knee, shoulder, elbow, forearm, chest, back and temporomandibular joints are implicated(1,2).

Disease Characteristics Fibroma of tendon sheath depicts a male predominance with a male to female proportion of $3: 1$. The tumefaction has a propensity for young to middle aged individuals and commonly appears betwixt $2^{\text {nd }}$ to $5^{\text {th }}$ decade, predominantly betwixt 13 years to 69 years, with an average age of disease incrimination at 40.9 years(1). Fibroma of tendon sheath involves the small joints as fingers and wrists in an estimated $80 \%$ to $85 \%$ individuals whereas incrimination of large joints is exceptional. Preponderantly, small joints are implicated wherein proportionate incrimination of tendons and tendon sheaths of fingers is at around $47.9 \%$, hands at nearly $24.8 \%$ and wrist at roughly $10.3 \%$. Large joints commonly incriminated with fibroma of tendon sheath is the knee joint wherein the posterior cruciate ligament and/or posterior capsule is implicated. Notwithstanding, fibroma of tendon sheath implicating large joints as the elbows, hips, shoulders, knees or ankles is exceptional and denominated in an estimated $2.8 \%$ to $4.2 \%$ instances $(1,2)$. Nevertheless, fibroma of tendon sheath implicates the knee $(74.4 \%)$, elbow $(11.6 \%)$, ankle $(9.3 \%)$ or shoulder $(4.7 \%)$ as a component of large joint incrimination. The neoplasm can adhere intensely to distal tendons of attributed skeletal muscles $(1,2)$.

Clinical Elucidation Fibroma of tendon sheath is a benign, exceptional, gradually progressive, neoplastic condition constituted by solitary, well circumscribed, lobulated accumulation of fibrocartilaginous tissue adherent to abutting tendon or tendon sheath. Typically, the neoplasm is discerned upon hands, wrists, fingers and uncommonly, the lower extremities. Volar aspect of thumb, index and middle finger are frequently implicated(3).

on sheath appears as a miniature, painless, gradually progressive nodule. The neoplasm can appear as an intra- articular lesion abutting synovial membrane or an extra- articular lesion. Fibroma of tendon sheath of large joints demonstrates symptoms such as pain (62.5\%), palpable mass or swelling (54.2\%) and decimated joint mobility $(50 \%)$. Joint effusion and locked joint appears exceptionally. Extra articular fibroma of tendon sheath of large joints depicts pain upon joint movement or infrequent, radiating pain due to irritation of soft tissue circumscribing the tumefaction $(3,4)$. Fibroma of tendon sheath is often associated with delayed clinical representation of a painless, gradually evolving tumefaction which can abut, irritate and compress encompassing soft tissues. An estimated one third (33\%) instances delineate minimal pain and soft tissue tenderness on account of compression of subjacent nerves, traversing beneath the neoplasm $(3,4)$. Fibroma of tendon sheath can engender "trigger wrist" due to tumour impingement upon adherent flexor tendons within the carpal canal with consequent occurrence of "snapping finger" or "carpal tunnel syndrome". Tumefaction can initiate complete limitation of finger flexion. An estimated beneath $<10 \%$ subjects demonstrate a history of trauma.

On histological examination, tendon sheath is typically layered with an epithelium in contiguity with extraneous fibrous tendon sheath and inner synovial sheath with embedded blood vessels $(4,5)$. As joint capsule morphologically resembles structure of tendon sheath, particularly where joint capsule is constituted by dual layer of dense, extraneous fibrous connective tissue and inmost synovial layer with ingrained vasculature, it can be surmised that fibroma of tendon sheath can arise from joint capsule, besides originating from the tendon or tendon sheath(4).

Histological Elucidation On gross examination, a whitish, well circumscribed, lobulated, fibrous nodule or a lesion encompassed with thin capsule is observed. Cut surface is firm, rubbery and greyish -white. Microscopically, the tumefaction is composed of spindle-shaped or stellate cells simulating fibroblasts. Cellular component is dispersed within a dense, collagenous stroma. Cellular or nuclear atypia and malignant metamorphoses is absent. Majority of lesions are hypo-cellular although certain neoplasms exhibit peripheral hyper-cellularity, akin to lesions of nodular fasciitis $(4,5)$. The tumefaction is comprised of dense, collagenous tissue demonstrating variable proportion of collagenisation and tumour cellularity. Peripheral neoplastic zone is markedly cellular and delineates slit- like, vascular spaces. Certain tumours can enunciate complete hyalinization. Mitotic activity is pertinent to cellularity of the neoplasm. Additionally, diverse inflammatory cells and myxoid stroma can be observed in specific instances. Giant cells or foamy cells are exceptional $(4,5)$.

Typically, fibroma of tendon sheath demonstrates elongated, thin- walled blood vessels or vascular clefts or slit- like vascular spaces. The minimally cellular neoplasm is composed of spindle-shaped cells intermingled within wavy fascicles of collagenous tissue and an admixture of slit- like blood vessels(5).

The tumefaction is enveloped with synovial membrane. Thus, it is surmised that intra articular fibroma of tendon sheath can originate from synovial membrane(5). 


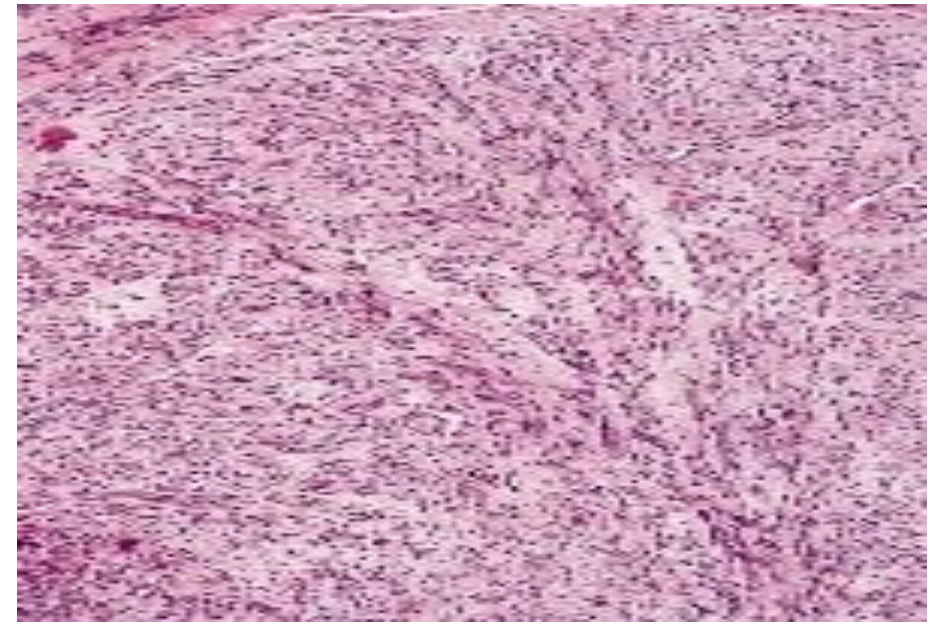

Figure 1 Fibroma of tendon sheath demonstrating fascicles of spindleshaped and stellate cells, foci of collagenous stroma and slit-like blood vessels(11).

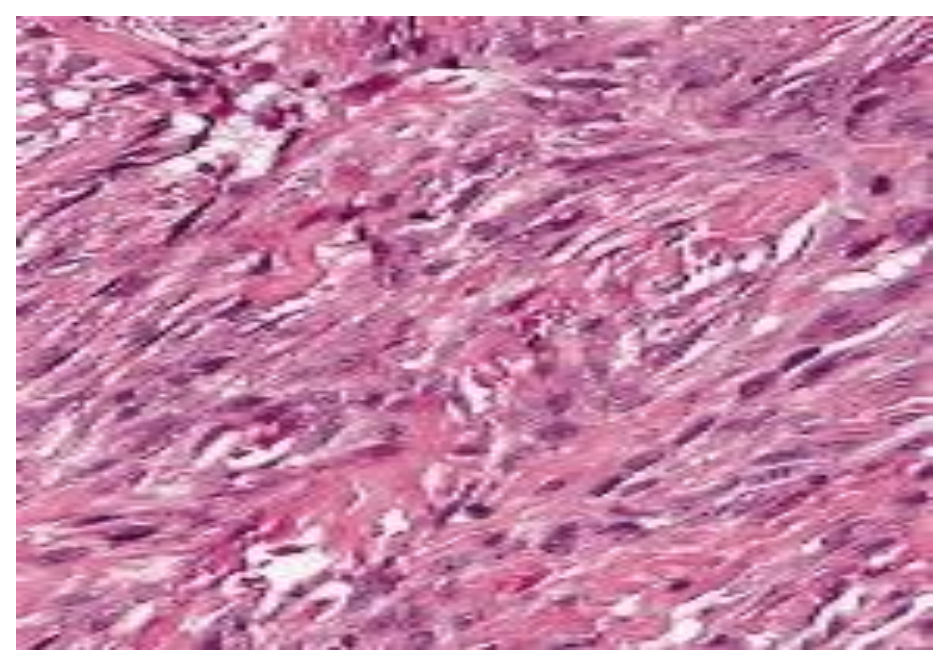

Figure 2 Fibroma of tendon sheath depicting strands and bundles of spindle-shaped cells with intermingled collagenous stroma and thinwalled blood vessels(11).

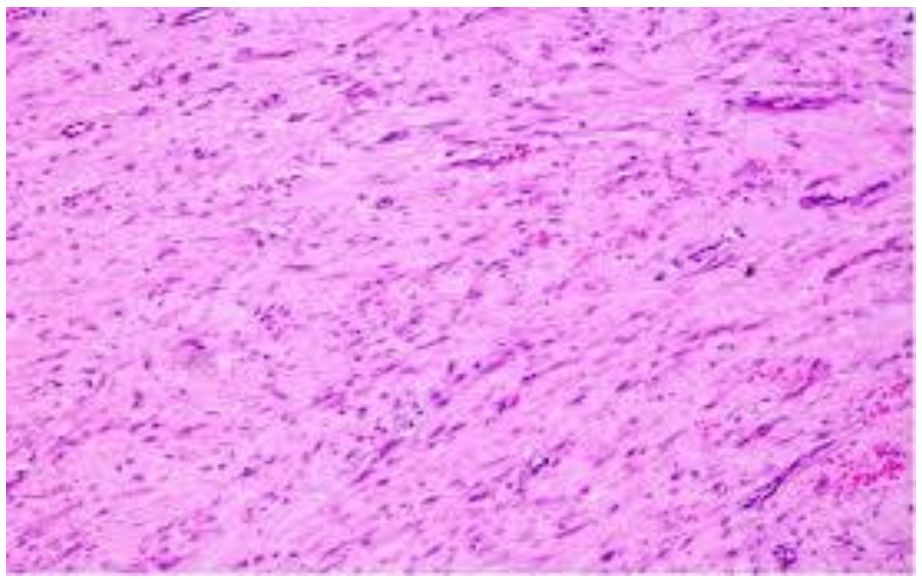

Figure 3 Fibroma of tendon sheath delineating bundles of spindle cells with attenuated blood vessels and collagen stroma along with an absence of giant cells(12).

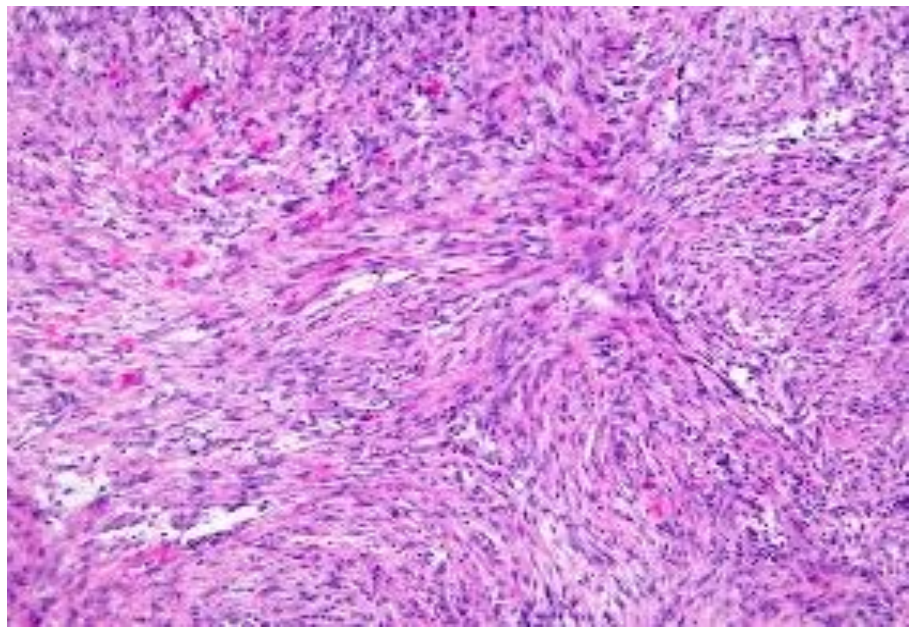

Figure 4 Fibroma of tendon sheath exhibiting interlacing strands of plump spindle-shaped cells with commingled slit-like vasculature and collagen stroma besides an absence of giant cells(13).

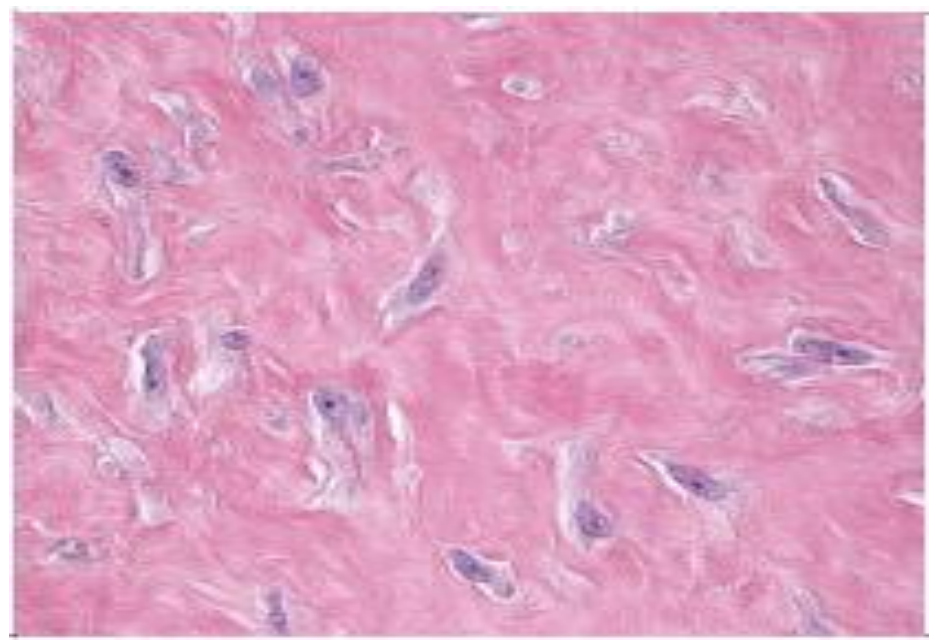

Figure 5 Fibroma of tendon sheath delineating fascicles and bands of plump, spindle-shaped and stellate cells with admixed attenuated vascular elements and collagen fibrils(14).

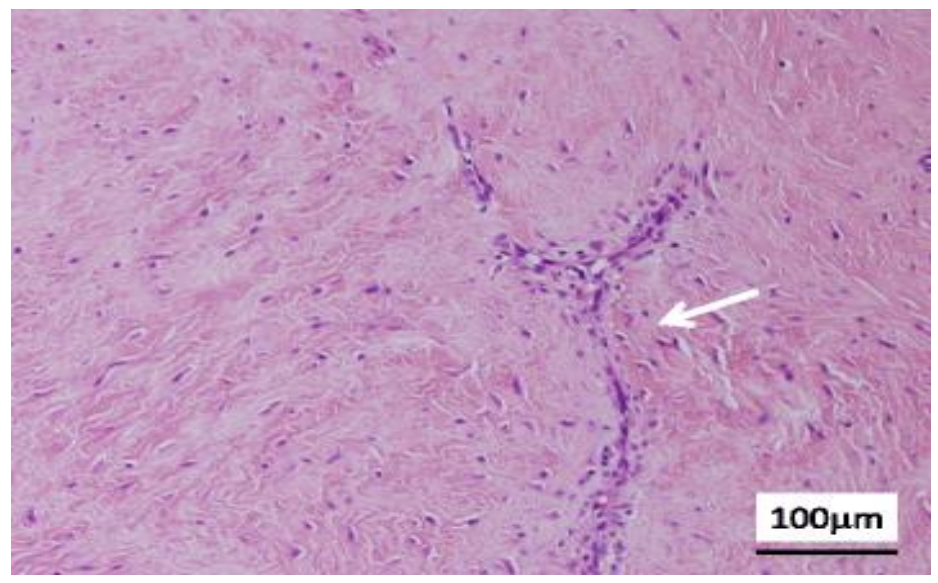

Figure 6 Fibroma of tendon sheath exemplifying strands and bundles of plump spindle-shaped cells, diverse slit-like vascular configurations and collagenous stromal articulations(15). 


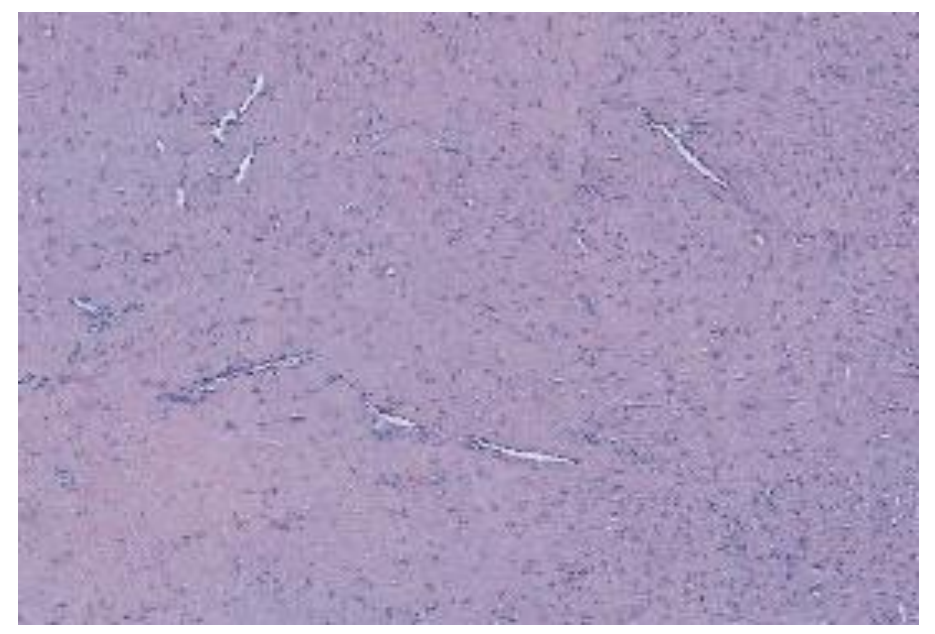

Figure 7 Fibroma of tendon sheath enunciating bands of stellate and spindle-shaped cells admixed with thin-walled vascular arrangements, collagen stroma and an absence of foamy macrophages(16).

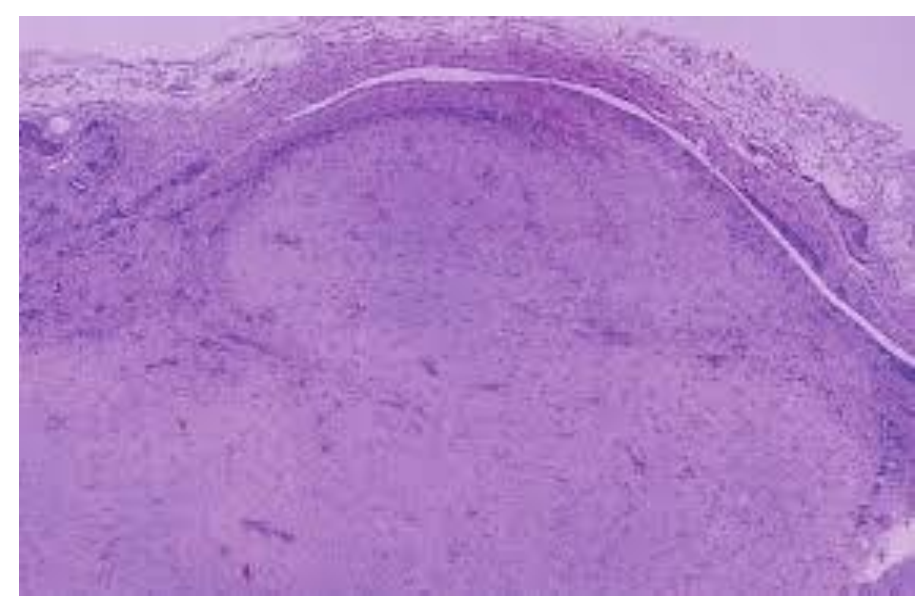

Figure 8 Fibroma of tendon sheath exhibiting nodules of bland, spindleshaped cells, intermixed collagenous stroma, thin-walled blood vessels and a limiting fibrous tissue capsule(17).

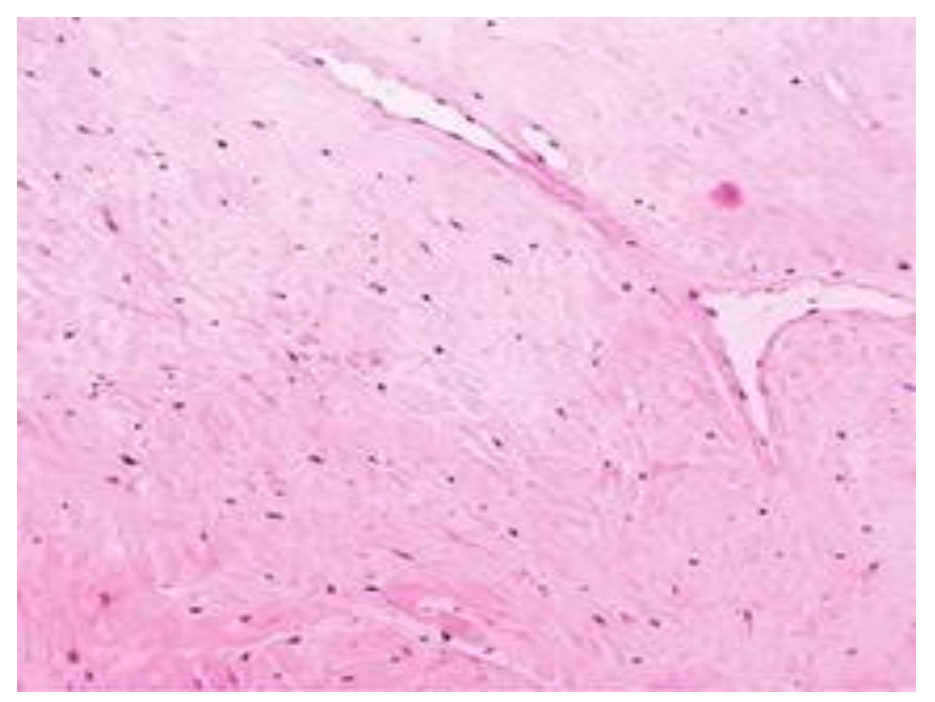

Figure 9 Fibroma of tendon sheath exemplifying slit-like vascular arrangements surrounded by lobules of plump, spindle-shaped cells and intermixed collagenous stroma(18).
Immune Histochemical Elucidation: Fibroma of tendon sheath is immune reactive to vimentin, muscle specific actin (MSA), smooth muscle actin (SMA), factor XIIIa and CD34. CD68 (+/-) may or may not be immune reactive whereas desmin is immune non reactive. On ultrastructural examination, predominant cell types configuring the neoplasm are myofibroblasts and fibroblasts. Myofibroblasts are predominant within cellular areas whereas fibroblasts are dominant within collagen-rich areas (6). On cytogenetic analysis, aberrant chromosomal complement can be discerned in fibroma of tendon sheath. Genomic deletions of chromosome 9 or additions in chromosome 16 (p11.2),(q24) and translocation $\mathrm{t}(11 ; 15)(\mathrm{q} 23 ; \mathrm{q} 22)$ are observed. Also, cytogenetic assay of fibroma of tendon sheath delineates genomic translocation with break point of long arm of chromosome 11. Genomic translocation betwixt chromosome $2 ; 11$ can be delineated $(5,6)$.

Differential Diagnosis Fibroma of tendon sheath requires a segregation from neoplasia such as schwannoma, chondroma or tenosynovial giant cell tumour. Exceptionally, haemangioma or tenosynovial fibroma can resemble fibroma of tendon sheath. Certain mesenchymal neoplasia are discerned at specific sites as soft tissues of hand or adjunctive locales. Thus, cogent soft tissue tumours as lipoma, lipofibromatous hamartoma, epidermal cyst, neuroma, angiomyolipoma, non-skeletal chondroma, synovial sarcoma, ganglion cyst, Dupuytren's contracture or fibromatosis necessitate a differentiation(4,6). Fibroma of tendon sheath mandates a demarcation from benign mesenchymal neoplasia as giant cell tumour of tendon sheath or localized nodular tenosynovitis, circumscribed fibromatosis, nodular fasciitis, neurofibroma, leiomyoma, scar tissue or benign fibrous histiocytoma(4,6). Fibroma of tendon sheath emerging within large joints as the knee joint mandates a distinction from giant cell tumour of tendon sheath or localized nodular tenosynovitis, pigmented villonodular synovitis, synovial chondromatosis or nodular fasciitis(5). Clinically, tenosynovial giant cell tumour is identical to fibroma of tendon sheath. Aforesaid neoplasms particularly arise within fingers, are attached to the tendon sheath, appear firm, well circumscribed, multi-lobulated, greyish/white and demonstrate identical imaging signals upon magnetic resonance imaging (MRI). Fibroma of tendon sheath can be distinguished from tenosynovial giant cell tumour by cogent morphology. Fibroma of tendon sheath and giant cell tumour of tendon sheath arise in identical locations as fingers, wrist and knee joint. Giant cell tumour of tendon sheath is a frequently discerned lesion, in contrast to fibroma of tendon sheath (6,7). However, aforesaid neoplasms demonstrate distinct histological features. Fibroma of tendon sheath is hypo-cellular and comprised of slit- like vascular configurations embedded within a dense, collagenous matrix. In contrast, giant cell tumour of tendon sheath is a cellular neoplasm displaying multiple multinucleated giant cells, foamy macrophages and haemosiderin pigment deposits, significant features for appropriate differentiation on histology $(6,7)$. Magnetic resonance imaging of giant cell tumour of tendon sheath demonstrates a solid, spheroidal, elliptical or multi-lobular soft tissue neoplasm abutting or situated within a joint space and is associated with minimal signal intensity upon T1 and T2 weighted images, akin to fibroma of tendon sheath. Contrast enhancement is usually prominent with giant cell tumour of tendon sheath whereas fibroma of tendon sheath is accompanied by an absence of or mildly enhanced uptake of contrast medium(6,7). Aforesaid neoplasms are efficaciously managed with localized tumour eradication or re- excision of the tumefaction. Nodular fasciitis is typically engendered from fascial surface, extends into circumscribing subcutaneous tissue and occasionally, skeletal muscle tissue. Thus, it is hypothesized that intra articular genesis of nodular fasciitis is 
infrequent(6,7). Clinically, nodular fasciitis represents as a rapidly evolving, painful tumefaction. Upon magnetic resonance imaging (MRI), majority of lesions of nodular fasciitis predominantly demonstrate minimal T1 imaging intensity and heterogeneously enhanced T2 signal intensities. Also, variable, perilesional, reticulated soft tissue oedema is cogitated upon acquisition of fluid- sensitive imaging $(7,8)$. Nodular fasciitis morphologically recapitulates fibroma of tendon sheath. However, characteristic vascular patterns visualized in fibroma of tendon sheath is absent in nodular fasciitis. Representation of a disorderly, "tissue culture" like tumour configuration, prominent myxoid stroma and foci of red cell extravasation are indicative of nodular fasciitis(7).

Investigative Assay Assessment of fibroma of tendon sheath on plain radiography is unremarkable. Computerized tomography (CT) of fibroma of tendon sheath is normal. Magnetic resonance imaging (MRI) is advantageous for categorising the neoplasm, tumour localization and evaluation of cogent imaging features. Fibroma of tendon sheath appears as a mass with well defined perimeter appearing superficial to distal tendon sheath. The mass is hypo-intense or isointense on T1 weighted imaging and appears hypo-intense or is accompanied by various signal intensities on $\mathrm{T} 2$ weighted imaging, in contrast to circumscribing skeletal muscle tissue $(7,8)$. Generally, tumours with minimal signal intensity on T2 weighted imaging are accompanied by significant quantities of fibrous tissue and are preponderantly hypo-cellular. Occasionally, centric, enhanced signal intensity with mildly reduced, peripheral signal intensity can be delineated on T2 weighted imaging on account of centroidal, hyper-cellular zone and myxoid alterations intermingled with slit- like, vascular elements within the tumefaction $(7,8)$.

Gadolinium enhanced magnetic resonance imaging (MRI) enunciates distinctive patterns of contrast enhancement, defined as rim or peripheral, patchy or focal, confined to the tumefaction and lobular enhancement. Peripheral image enhancement is contingent to vascular proliferation emerging at tumour periphery $(8,9)$. Image enhancement at tumour periphery appearing with an intra articular fibroma of tendon sheath indicates existence of synovium encompassing the tumour mass $(8,9)$.

Therapeutic Options Marginal resection of neoplasm with dissection through the pseudo-capsule and extermination of one centimetre to three centimetres of normal, uninvolved soft tissue or bony perimeter is a preferred therapeutic modality $(9,10)$. Neoplastic resection can be approached with arthroscopy. Alternatively, open surgical technique can be adopted for treating tumours exceeding > 3 centimetre magnitude with removal of an appropriate margin of normal or uninvolved soft tissue. The tumour is devoid of reoccurrence following adequate surgical eradication $(9,10)$.
Adequate treatment is comprised of localized tumour excision with preservation of pertinent anatomical structures. Tumour eradication can be challenging due to adherence to tendinous articulations wherein therapy is required to relieve clinical symptoms with functional preservation. Around one fourth $(25 \%)$ of tumefaction can reoccur following surgical extermination, probably due to inadequate eradication of tumour lobules during initial surgery. However, fibroma of tendon sheath is non- aggressive and the neoplasm does not demonstrate malignant metamorphoses, mitotic activity or distant metastasis $(9,10)$.

\section{References}

1. Geschickter CF, Copeland MM" Tumours of the Bone" Third Edition J.B.Lippincott; Philadelphia1949 pp693-695.

2. Chung EB, Enzinger FM " Fibroma of the tendon sheath" Cancer 1979:44(5);1945-1954.

3. Suzuki K, Yasuda T et al" Fibroma of tendon sheath around large joints - clinical characteristics and literature review"

4. Jordan MM, Accomazzo R et al "Fibroma of the tendon sheath arising from the flexor digitorum supericialis tendon " Eplasty 2019:19;ic3.

5. Magetsari RMSN, Irawan MNS" Surgery on aggressive fibroma of the posterior compartment of the knee- a case report" Int J Surg Case Rep 2019:65;115-118.

6. Fu Z, Liu J et al" Fibroma of the tendon sheath embedded in carpal bones mimicking carpal enchondroma- a case report" Medicine (Baltimore) 2019:98(16); e15262.

7. Al-Qattan MM" Fibroma of the tendon sheath- a series of 20 patients with 23 tumours" J Hand Surg Eur 2014:39(3);300-5.

8. Heckert R, Bear J et al" Fibroma of the tendon sheath - a rare hand tumour" Pol Przegl Chir 2012:84(12);651-6.

9. Park IJ, Lee YM et al "Multiple aetiologies of trigger wrist" $J$ Plast Reconstr Aesthet Surg 2016:69(3);335-40.

10. Ge Y, Guo G et al" Magnetic resonance imaging features of fibromas and giant cell tumours of the tendon sheathdifferential diagnosis" Eur Radiol 2019:29(7); 3441-3449

11. Image 1 and 2 Courtesy : Pathology Outlines

12. Image 3 Courtesy : Journal of Neoplasm.

13. Image 4 Courtesy : Twitter

14. Image 5 Courtesy : Basic Medical Key

15. Image 6 Courtesy : BMC Musculoskeletal disorders

16. Image 7 Courtesy : Wikipedia.

17. Image 8 Courtesy : Taylor and Francis Online

18. Image 9 Courtesy: Research gate 